\title{
ON UNIVALENT POLYNOMIALS
}

\author{
by DAVID A. BRANNAN
}

(Received 31 January, 1969)

1. Introduction. Let $P_{n}$ be the class of normalised polynomials of the form

$$
p_{n}(z)=z+a_{2} z^{2}+\ldots+a_{n} z^{n}
$$

of degree $n$ which are univalent in $U=\{|z|<1\}$. In this note we discuss the coefficients of polynomials in $P_{n}$ and in some of its subclasses.

Our principal tools will be

Lemma 1.1. (Dieudonné criterion) [6]. The polynomial $p_{n}(z)$, of the form (1.1), is univalent in $U$ if and only if the associated equation of $p_{n}(z)$,

$$
\phi(x, \theta)=1+\sum_{k=2}^{n} a_{k} x^{k-1} \sin (k-1) \theta / \sin \theta=0,
$$

has no roots in $|x|<1$, for any $\theta$ with $0 \leqq \theta \leqq \frac{1}{2} \pi$.

Lemma 1.2. (Cohn rule) [9]. Suppose that

$$
f(x)=a_{0}+a_{1} x+\ldots+a_{n} x^{n}
$$

is a polynomial of degree $n$, and

$$
f^{*}(x)=\bar{a}_{n}+\bar{a}_{n-1} x+\ldots+\bar{a}_{0} x^{n} .
$$

Then, if $\left|a_{0}\right| \geqq\left|a_{n}\right|$, the polynomial

$$
f_{1}(x)=\bar{a}_{0} f(x)-\bar{a}_{n} f^{*}(x)
$$

has the same number of zeros in $|x|<1$ as has $f(x)$.

Finally we recall the definitions of two classes of univalent functions which will appear later. The analytic function $f(z)$ is said to be starlike in $U$ if $f(0)=0$, and the segment $\left[0, f\left(z_{0}\right)\right]$ lies in $f(U)$ for any $z_{0}$ in $U[7]$; analytically this may be expressed by the condition

$$
\operatorname{Re}\left(z f^{\prime} \mid f\right)>0 \quad(z \text { in } U) .
$$

Further, the analytic function $g(z)$ is said to be close-to-convex in $U$ if $g(0)=0$, and

$$
\operatorname{Re}\left(z g^{\prime} i f\right)>0 \quad(z \text { in } U)
$$

for some starlike function $f(z)$ [8].

I would like to thank Professor J. Clunie for introducing me to the class $P_{n}$, and for his help and encouragement over a long period in this work. 
2. A particular subclass of $P_{n}$. If the polynomial $p_{n}(z)$, of the form (1.1), is univalent in $U, p_{n}^{\prime}(z)$ cannot vanish in $U$; consequently $\left|a_{n}\right| \leqq 1 / n$. In this section we consider polynomials in $P_{n}$ where $a_{n}=1 / n$.

Suppose that

$$
p_{n}(z)=z+a_{2} z^{2}+\ldots+a_{n-1} z^{n-1}+z^{n} j n .
$$

It was shown in [3] that, if $p_{n}(z)$ belongs to $P_{n}$, then

$$
(n-k) a_{n-k}=(k+1) \bar{a}_{k+1} \quad(1 \leqq k \leqq n-2) .
$$

In the opposite direction we have

TheOREM 2.1. Suppose that $p_{n}(z)$ is of the form (2.1). Then, if

$$
(n-k) a_{n-k}=(k+1) \bar{a}_{k+1} \quad(1 \leqq k \leqq n-2)
$$

and each $a_{k+1}$ is sufficiently small, $p_{n}(z)$ belongs to $P_{n}$.

Proof. The polynomial $p_{n}(z)$ belongs to $P_{n}$ if the equation

$$
1+\sum_{k=1}^{n-2} a_{k+1} \frac{\sin (k+1) \theta}{\sin \theta} x^{k}+\frac{\sin n \theta}{n \sin \theta} x^{n-1}=0
$$

has no roots in $|x|<1$ for $0 \leqq \theta \leqq \frac{1}{2} \pi$. Applying the Cohn rule to this equation, since $|\sin n \theta / n \sin \theta| \leqq 1$ for $0 \leqq \theta \leqq \frac{1}{2} \pi$, we see that $p_{n}(z)$ belongs to $P_{n}$ if

$$
\begin{aligned}
0 & =1-\left(\frac{\sin n \theta}{n \sin \theta}\right)^{2}+\sum_{k=1}^{n-2} x^{k}\left(a_{k+1} \frac{\sin (k+1) \theta}{\sin \theta}-\bar{a}_{n-k} \frac{\sin n \theta \sin (n-k) \theta}{n \sin \theta \sin \theta}\right) \\
& =1-\left(\frac{\sin n \theta}{n \sin \theta}\right)^{2}+\sum_{k=1}^{n-2} x^{k} a_{k+1}\left(\frac{\sin (k+1) \theta}{\sin \theta}-\frac{(k+1) \sin n \theta \sin (n-k) \theta}{(n-k) n \sin \theta \sin \theta}\right)
\end{aligned}
$$

has no roots in $|x|<1$ for $0 \leqq \theta \leqq \frac{1}{2} \pi$. Now each coefficient of $x^{r}(0 \leqq r \leqq n-2)$ has a double zero at $\theta=0$, and the constant term is always positive otherwise. Hence, if all the coefficients of $x^{5}$ are chosen sufficiently small, this equation has no roots in $|x|<1$, and $p_{n}(z)$ belongs to $P_{n}$.

In a much underestimated paper [1] Alexander showed that the polynomials $\sum_{k=1}^{n} z^{k} / k$ and $\sum_{k=0}^{n} z^{2 k+1} /(2 k+1)$ are univalent in $U$. We can put this result in a more general setting in

THEOREM 2.2. Suppose that

$$
p_{n}(z)=z+\sum_{k=2}^{n} a_{k} z^{k}, \quad \text { and } q_{n}(z)=z+\sum_{k=1}^{n} b_{2 k+1} z^{2 k+1},
$$

where $k a_{k}$ and $(2 k+1) b_{2 k+1}$ decrease as $k$ increases. Then $p_{n}(z)$ and $q_{n}(z)$ are close-to-convex univalent functions in $U$. 
Proof. We have, for $z \in U$,

$$
\begin{aligned}
\operatorname{Re}\left\{\frac{z p_{n}^{\prime}(z)}{z /(1-z)}\right\} & =\operatorname{Re}\left\{1+\left(1-2 a_{2}\right) z+\sum_{k=2}^{n-1}\left(k a_{k}-(k+1) a_{k+1}\right) z^{k}-n a_{n} z^{n}\right\} \\
& \geqq 1-\left(1-2 a_{2}\right)-\sum_{k=2}^{n-1}\left(k a_{k}-(k+1) a_{k+1}\right)-n a_{n} \\
& =0 .
\end{aligned}
$$

Hence $p_{n}(z)$ is close-to-convex in $U$. Similarly for $q_{n}(z)$.

However, in contrast with Theorems 2.1 and 2.2, we have the following surprising result for starlike polynomials

THEOREM 2.3. Suppose that $p_{n}(z)$, of the form (2.1), belongs to $P_{n}$. Then $p_{n}(z)$ is starlike in $U$ if and only if $a_{k}=0$ for $2 \leqq k \leqq n-1$.

Proof. If $a_{2}=a_{3}=\ldots=a_{n-1}=0$, it is easy to show that $p_{n}(z)$ is starlike in $U$. We therefore assume that $p_{n}(z)$ is starlike in $U$, and then show that this implies that $a_{k}=0$ for $2 \leqq k \leqq n-1$. Then

$$
\frac{1+2 a_{2} z+\ldots+z^{n-1}}{1+a_{2} z+\ldots+z^{n-1} / n}=\frac{p_{n}^{\prime}(z)}{h(z)}
$$

(where $h(z)=p_{n}(z) / z$ ) has positive real part in $U$. Since $p_{n}(z)$ belongs to $P_{n}$, we have that $(k+1) a_{k+1}=(n-k) \bar{a}_{n-k}$ for $1 \leqq k \leqq n-2$. Consequently, on $|z|=1$, we may define

$$
\begin{aligned}
\alpha(\theta) & =p_{n}^{\prime}\left(z^{2}\right) / z^{n-1} \quad\left(z=e^{i \theta}\right) \\
& =2\left[\cos (n-1) \theta+2\left|a_{2}\right| \cos \left\{(n-3) \theta-\phi_{2}\right\}+\ldots\right],
\end{aligned}
$$

where $\phi_{k}=\arg a_{k}$. Furthermore, we may define $\beta(\theta)$ and $\gamma(\theta)$ by

$$
\begin{aligned}
\frac{p_{n}^{\prime}\left(z^{2}\right)}{h\left(z^{2}\right)} & =\frac{p_{n}^{\prime}\left(z^{2}\right)}{z^{n-1}} / \frac{h\left(z^{2}\right)}{z^{n-1}} \\
& =\frac{\alpha(\theta)}{\beta(\theta)+i \gamma(\theta)}=\frac{\alpha(\theta) \beta(\theta)-i \alpha(\theta) \gamma(\theta)}{\beta^{2}(\theta)+\gamma^{2}(\theta)},
\end{aligned}
$$

where $z=e^{i \theta}$. No difficulty arises from the denominator, since the univalency of $p_{n}(z)$ ensures that

$$
\beta^{2}(\theta)+\gamma^{2}(\theta)=\left|h\left(e^{2 i \theta}\right)\right|^{2}=\left|p_{n}\left(e^{2 i \theta}\right)\right|^{2}>0
$$

for $0 \leqq \theta \leqq 2 \pi$. We now show that $\alpha(\theta)$ can have only simple zeros for $0 \leqq \theta \leqq 2 \pi$. Let $\phi$ be a zero of $\alpha(\theta)$. Now, with $z=e^{i \theta}$, we have

$$
\alpha^{\prime}(\theta)=\frac{\partial}{\partial \theta}\left[\frac{p_{n}^{\prime}\left(z^{2}\right)}{z^{n-1}}\right]=i z\left[\frac{2 z p_{n}^{\prime \prime}\left(z^{2}\right)}{z^{n-1}}-(n-1) \frac{p_{n}^{\prime}\left(z^{2}\right)}{z^{n}}\right] .
$$


Now $\alpha(\phi)=0$, so that $p_{n}^{\prime}\left(z^{2}\right)=0$ when $z=e^{i \phi}$. Hence, if $\alpha^{\prime}(\phi)$ is also zero, we see that $p_{n}^{\prime \prime}\left(z^{2}\right)$ is zero at $z=e^{i \phi}$ as well. But then $p_{n}^{\prime \prime}(z)$ is zero at $z=e^{2 l \phi}$. This, however, is impossible, since the existence of a double zero of $p_{n}^{\prime}(z)$ on $|z|=1$ is ruled out by the univalency of $p_{n}(z)$ in $U$.

Now, the condition $\operatorname{Re}\left(z p_{n}^{\prime} / p_{n}\right) \geqq 0$ in $U$ may be written in the form

$$
\alpha(\theta) \beta(\theta) \geqq 0 \text { for } 0 \leqq \theta \leqq 2 \pi ;
$$

since the zeros of $\alpha(\theta)$ are simple, this in turn shows that, whenever $\alpha(\theta)=0$, necessarily $\beta(\theta)=0$. Now all of its $2(n-1)$ zeros lie in $0 \leqq \theta \leqq 2 \pi$ (corresponding to the $n-1$ zeros of $p_{n}^{\prime}(z)$ all on $\left.|z|=1\right)$ in the case of $\alpha(\theta)$, and hence the same must be true of $\beta(\theta)$ since it is also a trigonometric polynomial of degree $n-1$. Since a polynomial which has its maximum number of zeros is determined by these zeros to within a constant factor, it follows that, for some constant $C$, we have

or

$$
\alpha(\theta)=C \beta(\theta)
$$

$$
p_{n}^{\prime}\left(z^{2}\right) / z^{n-1}=C \operatorname{Re}\left\{p_{n}\left(z^{2}\right) / z^{n-1}\right\} \text { on }|z|=1 \text {. }
$$

Expanding both sides of this equation, and equating the highest terms, with $z=e^{i \theta}$, we find that $C=2 n /(n+1)$. Substituting this value of $C$, and equating the other terms of the expansion in turn, we find that $a_{k}=0$ for $2 \leqq k \leqq n-1$. This completes the proof.

Note. In the case $n=3$, this result also appears in [4].

3. Some coefficient bounds for $P_{n}$. First we give bounds for the central coefficient of particular trinomials in $P_{2 n+1}$.

THEOREM 3.1. The polynomial

$$
p_{2 n+1}(z)=z+a z^{n+1}+z^{2 n+1} /(2 n+1)
$$

belongs to $P_{2 n+1}$ if and only if $a$ is real and

$$
|a| \leqq \operatorname{Min}_{\left(0, \frac{1}{2} \pi\right)}\left\{\frac{1+[\sin (2 n+1) \theta /(2 n+1) \sin \theta]}{|\sin (n+1) \theta / \sin \theta|}\right\}=\pi / 4 n\{1+o(1)\} \text { for large } n .
$$

Note. By (2.2), $a$ must be real, and so we may assume that $a \geqq 0$.

Proof. Applying the Cohn rule to the associated Dieudonne equation for $p_{2 n+1}(z)$, and using the fact that $|\sin (2 n+1) \theta /(2 n+1) \sin \theta|<1$ for $0<\theta \leqq \frac{1}{2} \pi$, the first inequality follows. Since $(2 / \pi) x \leqq \sin x \leqq x$ for $0 \leqq x \leqq \frac{1}{2} \pi$, we can show that the above minimum occurs in $0 \leqq \theta \leqq 4 \pi /(2 n+1)$; by elementary differentiation, it must occur at $\pi /(2 n+1)\{1+o(1)\}$ for large $n$. This gives the last inequality.

COROLlaRY. The polynomials $z+a z^{2}+\frac{1}{3} z^{3}$ and $z+b z^{3}+\frac{1}{5} z^{5}$ are univalent in $U$ if and only if $a$ and $b$ are real, and $|a| \leqq 8 / 9$ and $|b| \leqq 3 / 5$.

We now turn to the estimation of the $(n-1)$ th coefficients of polynomials in $P_{n}$. 
THEOREM 3.2. Suppose that $p_{n}(z)$, of the form (1.1), belongs to $P_{n}$. Then

$$
(n-1)\left|a_{n-1}\right| \leqq 1+2 n\left|a_{2} a_{n}\right|-n^{2}\left|a_{n}\right|^{2}<4 ;
$$

in particular,

$$
(n-1)\left|a_{n-1}\right| \leqq\left\{\begin{array}{l}
1+\left|a_{2}\right|^{2}, \text { if }\left|a_{2}\right| \geqq 1, \\
2\left|a_{2}\right|, \text { if }\left|a_{2}\right|<1 .
\end{array}\right.
$$

Proof. By the Dieudonné criterion, since $p_{n}(z)$ belongs to $P_{n}$, the equation

$$
1+\sum_{k=1}^{n-1} a_{k+1} \frac{\sin (k+1) \theta}{\sin \theta} x^{k}=0
$$

has no roots in $|x|<1$ for $0 \leqq \theta \leqq \frac{1}{2} \pi$, and $\left|a_{n}\right| \leqq 1 / n$. Applying the Cohn rule, we deduce that the equation

$$
1-\left|a_{n}\right|^{2}\left(\frac{\sin n \theta}{\sin \theta}\right)^{2}+\sum_{k=1}^{n-2} x^{k}\left(a_{k+1} \frac{\sin (k+1) \theta}{\sin \theta}-a_{n} \bar{a}_{n-k} \frac{\sin n \theta \sin (n-k) \theta}{\sin \theta \sin \theta}\right)=0
$$

has no roots in $|x|<1$ for $0 \leqq \theta \leqq \frac{1}{2} \pi$. Consequently

$$
\begin{aligned}
1-\left|a_{n}\right|^{2}\left(\frac{\sin n \theta}{\sin \theta}\right)^{2} & \geqq\left|a_{n-1} \frac{\sin (n-1) \theta}{\sin \theta}-a_{n} \bar{a}_{2} \frac{\sin n \theta \sin 2 \theta}{\sin \theta \sin \theta}\right| \\
& \geqq\left|a_{n-1} \frac{\sin (n-1) \theta}{\sin \theta}\right|-\left|a_{n} a_{2} \frac{\sin n \theta \sin 2 \theta}{\sin \theta \sin \theta}\right| ;
\end{aligned}
$$

substituting $\theta=0$, we obtain

$$
1-n^{2}\left|a_{n}\right|^{2} \geqq(n-1)\left|a_{n-1}\right|-2 n\left|a_{2} a_{n}\right| .
$$

This gives the first inequality. The next three follow at once by considering the behaviour of the expression $1+\left(n\left|a_{n}\right|\right)\left(2\left|a_{2}\right|\right)-\left(n\left|a_{n}\right|\right)^{2}$ where $\left|a_{2}\right| \leqq 2$ and $\left|a_{n}\right| \leqq 1 / n$.

Note 1. Suffridge [10] has shown that the polynomial

$$
\sum_{k=1}^{n} \frac{n-k+1}{n} \cdot \frac{\sin (k \pi / n+1)}{\sin (\pi / n+1)} z^{k}
$$

belongs to $P_{n}$. Consequently the constant 4 in (3.1) cannot be improved independently of $n$.

Note 2. Recent work has determined the coefficient regions for $P_{3}[3,5]$, for starlike polynomials in $P_{3}$ [4], and for the subclass of $P_{n}$ with real Maclaurin coefficients [10]. However, much work remains to be done on the general coefficient problem for $P_{n}(n>3)$. 
Note 3. Results similar to Theorems 2.1, 2.3, 3.1, and 3.2 were obtained in [2] for the class of " pseudo-polynomials"

$$
\mu_{n}(z)=z^{-1}+\sum_{k=1}^{n} a_{k} z^{k}
$$

analytic and univalent in $0<|z|<1$.

Note 4. Using the fact that the class of linearly-accessible functions of Biernacki is exactly the class of close-to-convex functions of Kaplan, we may observe that Alexander [1] showed that the polynomial

$$
p(z)=\int_{0}^{z}\left(1-e^{i \theta_{1}} t\right) \ldots\left(1-e^{i \theta_{n-1}} t\right) d t
$$

where $0 \leqq \theta_{1}<\theta_{2}<\ldots<\theta_{n-1}<2 \pi \leqq \theta_{n}=\theta_{1}+2 \pi$, is close-to-convex in $U$ if and only if

$$
\theta_{j+1}-\theta_{j} \geqq 2 \pi /(n+1) \quad(1 \leqq j \leqq n-1) \text {. }
$$

\section{REFERENCES}

1. J. W. Alexander, Functions which map the interior of the unit circle upon simple regions, Annals of Math. 17 (1915), 12-22.

2. D. A. Brannan, On univalent polynomials and related classes of functions, Thesis, University of London (1967).

3. D. A. Brannan, Coefficient regions for univalent polynomials of small degree, Mathematika 14 (1967), 165-169.

4. D. A. Brannan and L. Brickman, Coefficient regions for starlike polynomials; to appear.

5. V. F. Cowling and W. C. Royster, Domains of variability for univalent polynomials, Proc. Amer. Math. Soc. 19 (1968), 767-772. 78-81.

6. J. Dieudonné, Sur le rayon d'univalence des polynomes, C. R. Acad. Sci. Paris 192 (1931),

7. W. K. Hayman, Multivalent functions, Cambridge University Press (1958).

8. W. Kaplan, Close-to-convex schlicht functions, Michigan Math. J. 1 (1952), 169-185.

9. M. Marden, The geometry of the zeros of a polynomial in a complex variable, New York (1949).

10. T. J. Suffridge, On univalent polynomials, J. London Math. Soc. (2) 44 (1969), 496-504.

UNIVERSITY OF GLASGOW

Glasgow, W.2 